\title{
A pan-cancer perspective of matrix metalloproteases (MMP) gene expression profile and their diagnostic/prognostic potential
}

Emily Gobin ${ }^{\dagger}$, Kayla Bagwell ${ }^{\dagger}$, John Wagner ${ }^{\dagger}$, David Mysona, Sharmila Sandirasegarane, Nathan Smith, Shan Bai, Ashok Sharma, Robert Schleifer and Jin-Xiong She*

\begin{abstract}
Implication: By understanding Matrix Metalloprotease (MMP) dysregulation from a pan-cancer perspective, this study sheds light on the diagnostic potentials of MMPs across multiple neoplasms.

Background: MMPs are intriguing genes related to cancer disease progression, functional promotion of angiogenesis, invasion, metastasis, and avoidance of immune surveillance. Many studies have noted these genes are frequently upregulated in cancer. However, expression patterns of all MMPs and their diagnostic and prognostic potential have not been investigated in a pan-cancer perspective.

Methods: The Cancer Genome Atlas (TCGA) data were used to evaluate diagnostic and prognostic potential of 24 MMPs in fifteen different cancer types. Gene expression measured by RNA-seq was analyzed by differential expression, hierarchical clustering, and ROC analysis for individual genes and in combination.

Results: MMP1, MMP9, MMP10, MMP11, and MMP13 were almost universally upregulated across all cancers, with significant $(p<0.05)$ fold change ( $F C>2)$ in ten of fifteen cancers. MMP3, MMP7, MMP12 and MMP14) are significantly up-regulated in at least 10 cancer types. Interestingly, MMP2, MMP7, MMP23B, MMP27 and MMP28) are significantly down-regulated in seven to nine cancer types. Multiple MMPs possess AUC's > 0.9 in more than one cancer. However, survival analyses suggest that the prognostic value of MMPs is limited to clear cell renal carcinoma.

Conclusions: Most MMPs have consistently increased gene expression across cancers, while several MMPs have consistently decreased expression in several cancer types. Many MMPs have diagnostic value individually or in combination, while the prognostic value of MMPs is restricted to one subtype of kidney cancer.
\end{abstract}

Keywords: Gene expression, MMPs, Matrix metalloproteases, Biomarkers, Survival, Diagnosis, Prognosis, TCGA

\section{Background}

MMPs have been extensively studied for nearly 40 years and originally were noted for their role in degrading the extracellular matrix (ECM) [1]. Structurally, MMPs contain similar catalytic inhibitory domains. The catalytic domains add specificity to the target for degradation of

\footnotetext{
* Correspondence: jshe@augusta.edu

†Emily Gobin, Kayla Bagwell and John Wagner contributed equally to this work.

Center for Biotechnology and Genomic Medicine, Medical College of Georgia, Augusta University, Augusta, GA 30912, USA
}

each MMP. Traditional classification of MMPs delineates based on the first identified target of degradation. Over the years, MMPs have been found to play a remarkable number of regulatory roles at the cellular level in pathways such as apoptosis, immunity, cellular migration, and angiogenesis [2]. MMP functionality often complements classical tumor properties leading to invasion, immune system avoidance, and metastasis. Given these qualities, MMPs play a major role in carcinogenesis [1-4]. Table 1 provides an overview of specific role of each MMP in cancer. 
Table 1 MMPs in Cancer

\begin{tabular}{|c|c|}
\hline MMP & Role in Cancer \\
\hline \multicolumn{2}{|c|}{ Collagenases } \\
\hline 1 & Initial invasion, promotes metastasis $[5,6]$ \\
\hline \multicolumn{2}{|l|}{8} \\
\hline 13 & $\begin{array}{l}\text { Growth, invasion, and angiogenesis of skin squamous cell } \\
\text { carcinoma [7] }\end{array}$ \\
\hline \multicolumn{2}{|c|}{ Matrilysins } \\
\hline 7 & $\begin{array}{l}\text { Contributes to invasive potential, proliferation, anti-apoptotic, } \\
\text { immune surveillance }[1,8]\end{array}$ \\
\hline 26 & $\begin{array}{l}\text { Activates MMP- } 9 \text { in prostate cancer, role in early skin } \\
\text { carcinogenesis }[9,10]\end{array}$ \\
\hline \multicolumn{2}{|c|}{ Metalloelastase } \\
\hline 12 & Protective inhibition of tumor growth, anti-angiogenic [11] \\
\hline \multicolumn{2}{|c|}{ Stromelysins } \\
\hline 3 & $\begin{array}{l}\text { Invasion, metastasis, and epithelial to mesenchymal transition } \\
\text { [12-14] }\end{array}$ \\
\hline 10 & $\begin{array}{l}\text { Invasion, migration, and growth; prevents tumor cell apoptosis; } \\
\text { produces angiogenic and metastatic factors [15-17] }\end{array}$ \\
\hline 11 & $\begin{array}{l}\text { Produced by peritumoral stromal fibroblasts; regulates early } \\
\text { tumor invasion, implantation, and expansion; prevents apoptosis } \\
\text { of early cancer cells [18-20] }\end{array}$ \\
\hline \multicolumn{2}{|c|}{ Gelatinases } \\
\hline 2 & $\begin{array}{l}\text { Proteolytic degradation of extracellular proteins in tumor } \\
\text { invasion, collagenolytic pathway driver for lymphatic vessel } \\
\text { formation, tumor angiogenesis }[1,15,16]\end{array}$ \\
\hline 9 & $\begin{array}{l}\text { Proteolytic degradation of extracellular proteins during tumor } \\
\text { invasion }[1,15]\end{array}$ \\
\hline \multicolumn{2}{|c|}{ Enamelysin } \\
\hline 20 & Synthesized in odontogenic tumors [21] \\
\hline \multicolumn{2}{|c|}{ Membrane-Type } \\
\hline 14 & $\begin{array}{l}\text { Cleaves other pro MMPs (mainly MMP2) to activate them, role in } \\
\text { invasive blood vessel growth, and promoting metastasis. In vitro } \\
\text { has been shown to promote invasion }[22,23]\end{array}$ \\
\hline 15 & $\begin{array}{l}\text { In vitro shown to play role in epithelial to mesenchymal } \\
\text { transition, promotes angiogenesis }[24,25]\end{array}$ \\
\hline 16 & In vitro promotes invasion and metastasis $[26,27]$ \\
\hline 17 & Induce angiogenesis promote growth and metastasis $[25,28]$ \\
\hline 24 & $\begin{array}{l}\text { Progression in brain tumors, aides in migration and metastasis } \\
{[29,30]}\end{array}$ \\
\hline 25 & In vitro tumor growth promoter [31] \\
\hline \multicolumn{2}{|c|}{ Other } \\
\hline 19 & In vitro modulates proliferation, adhesion, and metastasis $[32,33]$ \\
\hline 21 & Expression changes associated with cancer prognosis. [34] \\
\hline $23 \mathrm{~A}$ & $\begin{array}{l}\text { Expression levels altered in multiple cancers. Urinary levels } \\
\text { decreased in renal cell carcinoma. }[35,36]\end{array}$ \\
\hline \multicolumn{2}{|l|}{$23 B$} \\
\hline \multicolumn{2}{|l|}{27} \\
\hline 28 & $\begin{array}{l}\text { Promotes epithelial to mesenchymal transition, promotes } \\
\text { invasion and metastasis [37] }\end{array}$ \\
\hline
\end{tabular}

Dysregulation of MMP expression has been noted in numerous studies at the protein and RNA levels in many cancer types (Table 1). Frequently, MMP dysregulation associates with prognostic differences, a trend noted in breast, ovarian, and colon cancers $[4,31]$. This manuscript uses data from The Cancer Genome Atlas (TCGA) to examine differences in RNA expression of MMPs in a comprehensive manner. By investigating data from fifteen TCGA cancer types, we seek to: 1) identify patterns of MMP dysregulation, 2) associate MMP expression to patient survival in all 15 cancer types, and 3) integrate the TCGA data with previously published data to gain pertinent insight regarding MMP on tumorigenesis and prognosis.

\section{Methods}

TCGA datasets

The TCGA gene expression (RNAseq) data (IlluminaHiSeq: $\log 2$-normalized_count +1 ) was downloaded from Xena browser (https://xenabrowser.net/datapages/). The fifteen cancer types selected had at least ten patients with adjacent normal samples. The cancer types are denoted by their TCGA four-letter abbreviation (See Abbreviations). Statistical analyses were performed to compare and contrast the expression levels of $24 \mathrm{MMP}$ genes.

\section{Statistical analyses}

All statistical analyses were performed using the $R$ language and environment for statistical computing ( $R$ version 3.2.2; $\mathrm{R}$ Foundation for Statistical Computing; www.r-project.org). The normalized counts were $\log 2$ transformed prior to all statistical analyses to achieve a normal distribution. Expression differences between cancer patients and adjacent normals were initially examined using a t-test where the amount and significance of change were depicted using fold change (FC: Cancer vs Adjacent Normal) and $p$-value (pval). Fold change was calculated as the median gene expression level. Trends in expression differences were identified using unsupervised clustering. Diagnostic power of expression differences (Cancer vs. Adjacent Normal) of individual, or combinations of, MMPs was assessed using the area under the curve (AUC) of the receiver operating characteristic (ROC). Sensitivity values were calculated at various specificity thresholds (90\%; 95\%; 99\%; 100\%). Cox proportional hazards models were used to evaluate the association of gene expression levels on overall survival (diagnosis to date of death). Survival data was obtained from the TCGA patient phenotype files. Patients who at the time of analysis were alive with no evidence of disease were censored at the date of last follow-up visit. Kaplan-Meier survival analysis and the log-rank test were used to compare survival differences in groups 
separated by median expression level at multiple thresholds. Each cancer was considered individually. Multivariate analysis was performed to identify combinations of proteins that had significant findings in the univariate analysis. Data was visualized using $\mathrm{R}$ software and Tableau 10.4 (Tableau Software, www. tableau.com).

\section{Results}

\section{MMP expression difference between Cancer and Normal}

Gene expression differences for the twenty-four MMP family members were analyzed in fifteen different cancer types in the Cancer Genome Atlas (TCGA) (Fig. 1). The most prevalent gene expression changes were upregulation as opposed to downregulation in tumor tissue versus control tissue. MMP subtypes had similarities in expression patterns, with dramatic upregulation in cancer for the majority of collagenases, matrilysins, metalloelastase, and stromelysins. Other MMPs that showed significant differences were strong upregulation of MMP14 and downregulation of MMP23B, MMP27, and $M M P 28$. The most significant dysregulations were among collagenases and stromelysins. MMP11, a stromelysin, was significantly upregulated in all cancer types except $\mathrm{KICH}$ and UCEC ( $p=1 \mathrm{E}-07$ to $1 \mathrm{E}-122)$. The other stromelysins, $M M P 3$ and $M M P 10$, were significantly upregulated in 7 cancer types and 10 cancer types respectively. $M M P 9$, a gelatinase, was significantly upregulated in twelve of the fifteen analyzed cancer types ( $\mathrm{p}=1 \mathrm{E}-05$ to $4 \mathrm{E}-27)$. Collagenases, $M M P 1$ and
$M M P 13$, were significantly altered in 11 cancer types and 12 cancer types, respectively ( $p=5 \mathrm{E}-05$ to $1 \mathrm{E}-77$ ).

Across cancer types, lung squamous (LUSC) and uterine corpus endometrial (UCEC) were distinct from the other cancer types with several significantly downregulated MMPs (Fig. 1). Additionally, renal cancers comparatively lacked significant upregulation of MMPs compared to adjacent normal tissue. This pattern is most noticeable among collagenases and stromelysins other than MMP11.

\section{MMP expression is heterogeneous across cancer types}

Expression of MMPs has a large degree of heterogeneity across cancers (Fig. 2). Some MMPs had very high numbers of transcripts present (gene cluster A), while six MMPs in gene cluster C (MMP27, MMP21, MMP20, $M M P 26, M M P 23 A$, and $M M P 8)$ featured scant numbers of transcript copies across any cancer tissue (Fig. 2a and b). The cancer types with the most significant changes in MMP gene expression were LUSC and HNSC (19 and 15 MMPs, respectively), and patients from these cancers generally clustered together upon unsupervised hierarchical clustering as seen in cluster 2 of Fig. 2a. This grouping in cluster 2 features high expression of gene cluster B genes (MMP3, MMP10, MMP13, MMP1, and $M M P 12$ ) in a pattern that is relatively unique among cancer types.

Among the high abundance MMPs, MMP9, MMP11, $M M P 14$, and $M M P 2$ had marked differences in expression among the three patient clusters. These genes were

\begin{tabular}{|c|c|c|c|c|c|c|c|c|c|c|c|c|c|c|c|c|}
\hline Gene & BLCA & BRCA & COAD & ESCA & HNSC & $\mathrm{KICH}$ & KIRC & KIRP & LIHC & LUAD & LUSC & PRAD & STAD & THCA & UCEC & Fold Change: \\
\hline MMP1 & 6.97 & 30.82 & 12.82 & 29.05 & 43.82 & 0.30 & 1.67 & 0.43 & 3.07 & 25.20 & 36.41 & 0.72 & 9.53 & 4.97 & 14.15 & $>4$ \\
\hline MMP8 & 1.72 & 1.45 & 3.51 & 3.10 & 2.63 & 0.89 & 1.20 & 1.35 & 0.74 & 1.84 & 1.96 & 1.04 & 3.14 & 1.33 & 1.47 & $2-4$ \\
\hline MMP13 & 7.88 & 55.31 & 8.79 & 27.54 & 53.93 & 2.20 & 1.89 & 1.13 & 1.38 & 29.46 & 25.82 & 0.74 & 5.73 & 4.07 & 1.98 & $0.5-2$ \\
\hline MMP7 & 3.19 & 0.44 & 141.85 & 14.07 & 4.14 & 0.07 & 0.26 & 2.48 & 0.31 & 2.13 & 1.65 & 0.96 & 13.11 & 5.01 & 1.84 & $0.5-0.25$ \\
\hline MMP26 & 1.02 & 1.06 & 1.03 & 1.00 & 1.01 & 4.36 & 1.06 & 1.07 & 1.01 & 1.02 & 1.02 & 6.22 & 1.05 & 1.03 & 0.13 & $<0.25$ \\
\hline MMP12 & 2.19 & 2.29 & 2.36 & 38.96 & 15.58 & 1.76 & 1.32 & 1.08 & 4.24 & 17.07 & 52.46 & 1.55 & 8.93 & 1.83 & 19.18 & pval >0.05 \\
\hline MMP3 & 0.69 & 4.83 & 23.03 & 28.61 & 15.55 & 1.10 & 1.00 & 0.99 & 1.31 & 5.83 & 24.65 & 0.95 & 13.69 & 1.58 & 3.36 & \\
\hline MMP10 & 2.21 & 5.72 & 6.17 & 20.48 & 11.38 & 1.04 & 1.17 & 0.90 & 2.15 & 5.13 & 34.32 & 4.24 & 6.21 & 2.13 & 5.10 & MMP Subtype \\
\hline MMP11 & 14.93 & 56.55 & 13.39 & 23.63 & 25.64 & 1.32 & 4.71 & 5.79 & 9.55 & 35.62 & 43.04 & 2.06 & 12.47 & 6.67 & 1.93 & Collagenases \\
\hline MMP2 & 0.34 & 0.78 & 0.89 & 1.08 & 2.58 & 0.70 & 1.07 & 0.44 & 0.80 & 1.06 & 0.77 & 0.60 & 1.11 & 0.95 & 0.30 & Matrilysins \\
\hline MMP9 & 3.59 & 6.88 & 2.01 & 10.01 & 17.19 & 3.24 & 9.90 & 3.77 & 2.88 & 4.80 & 4.19 & 3.98 & 5.83 & 1.72 & 7.92 & Metalloelastase \\
\hline MMP20 & 1.11 & 0.73 & 1.35 & 1.46 & 1.25 & 1.47 & 0.72 & 0.49 & 1.16 & 1.09 & 1.82 & 1.00 & 1.06 & 1.01 & 1.17 & Stromelysins \\
\hline MMP14 & 1.73 & 2.00 & 2.11 & 2.96 & 2.74 & 0.77 & 2.66 & 2.84 & 1.86 & 1.88 & 2.60 & 0.55 & 1.87 & 1.66 & 0.69 & Gelatinases \\
\hline MMP15 & 1.89 & 1.17 & 0.61 & 0.74 & 1.30 & 2.28 & 0.80 & 1.89 & 0.89 & 1.78 & 0.63 & 1.14 & 1.42 & 0.69 & 3.05 & Enamelysin \\
\hline MMP16 & 0.29 & 0.71 & 0.87 & 0.89 & 2.24 & 1.72 & 4.94 & 0.88 & 0.84 & 1.67 & 1.04 & 0.42 & 1.20 & 4.57 & 0.27 & Membrane-Type \\
\hline MMP17 & 0.74 & 1.43 & 0.96 & 1.85 & 2.36 & 0.70 & 2.09 & 1.60 & 1.25 & 4.40 & 2.02 & 0.80 & 0.92 & 0.80 & 0.44 & Other \\
\hline MMP24 & 1.04 & 0.74 & 0.73 & 0.69 & 0.72 & 3.15 & 1.00 & 1.67 & 1.16 & 0.68 & 0.25 & 1.09 & 0.93 & 1.04 & 1.04 & \\
\hline MMP25 & 1.04 & 1.56 & 0.33 & 1.80 & 1.60 & 0.88 & 3.61 & 1.74 & 0.78 & 0.58 & 0.39 & 1.23 & 1.12 & 0.81 & 1.37 & \\
\hline MMP19 & 0.79 & 0.55 & 1.12 & 0.66 & 1.94 & 0.50 & 1.73 & 1.68 & 0.88 & 0.37 & 0.26 & 0.90 & 1.26 & 0.64 & 0.45 & \\
\hline MMP21 & 0.89 & 0.85 & 0.97 & 0.61 & 0.97 & 0.61 & 1.24 & 0.85 & 1.30 & 0.89 & 0.83 & 0.93 & 0.82 & 0.72 & 0.78 & \\
\hline MMP23A & 0.61 & 0.93 & 0.96 & 0.88 & 1.20 & 0.78 & 1.10 & 0.78 & 0.99 & 0.83 & 0.42 & 0.92 & 1.30 & 1.10 & 0.74 & \\
\hline MMP23B & 0.19 & 0.59 & 0.53 & 0.64 & 1.47 & 0.18 & 0.70 & 0.54 & 0.52 & 0.51 & 0.27 & 0.78 & 0.73 & 1.04 & 0.45 & \\
\hline MMP27 & 0.28 & 0.18 & 0.27 & 0.51 & 0.27 & 1.01 & 1.05 & 0.96 & 1.03 & 0.93 & 1.26 & 0.98 & 0.52 & 0.85 & 0.56 & \\
\hline MMP28 & 0.57 & 0.25 & 0.09 & 0.60 & 1.91 & 0.14 & 0.86 & 0.87 & 1.29 & 0.16 & 0.08 & 0.53 & 0.30 & 0.70 & 0.10 & \\
\hline
\end{tabular}

Fig. 1 Differential gene expression of 24 matrix metalloproteinases (MMPs) in 15 different cancer types. Fold change and $p$-values shown were obtained through comparison of unmatched control tissue ( $\mathrm{N}$ between 11 and 114 ) to tumor tissue ( $\mathrm{N}$ between 66 and 1097 ). Fold change was calculated as the median expression of a gene in tumor divided by the median gene expression in adjacent normal tissue 


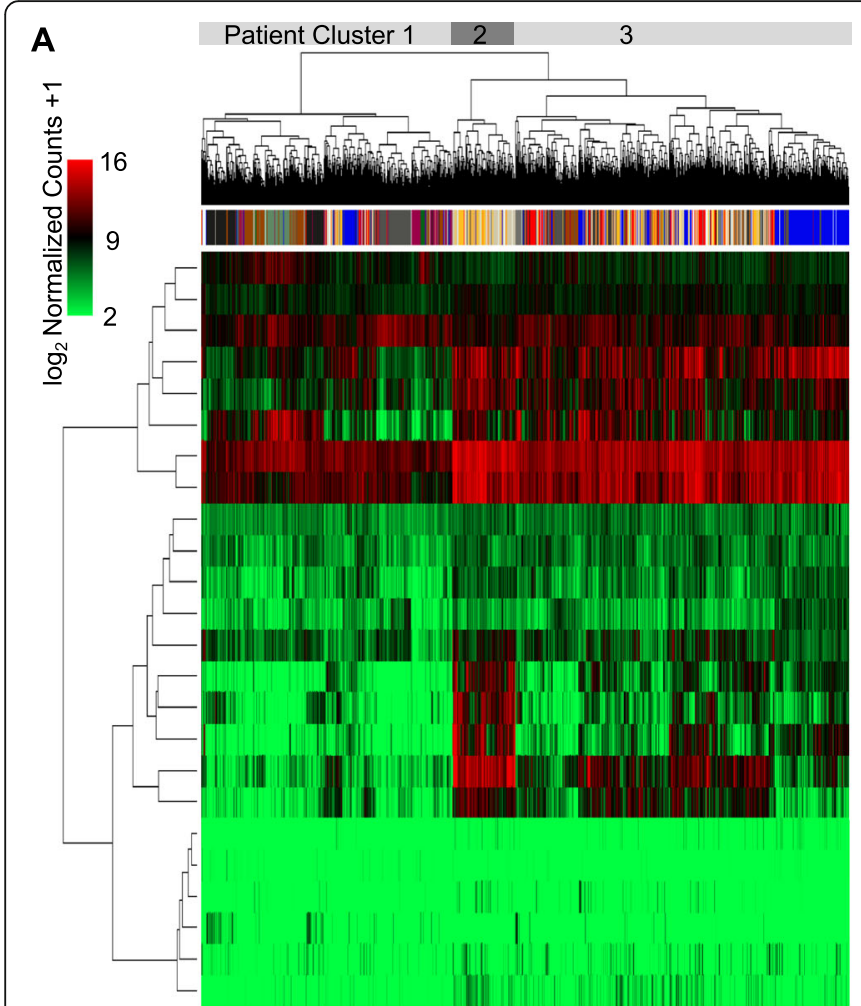

B

TCGA Cancer Tissue Site

$\mathrm{BLCA} \backsim \mathrm{ESCA} \backsim \mathrm{KIRC} \backsim \mathrm{LUAD} \backsim \mathrm{STAD}$

- BCRA $\square \mathrm{HNSC} \backsim \mathrm{KIRP} \square \mathrm{LUSC} \backsim$ THCA

$\because \mathrm{COAD} \approx \mathrm{KICH} \backsim \mathrm{LIHC} \backsim \mathrm{PRAD} \backsim \mathrm{UCEC}$

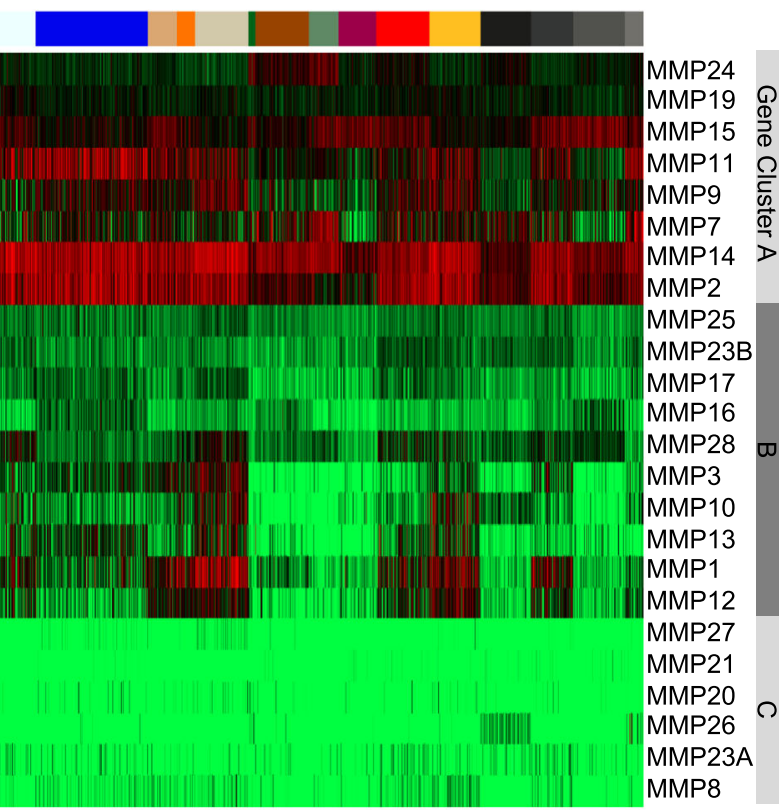

Fig. 2 Heat map representing color coded expression levels of 24 differentially expressed MMP genes in 15 different cancer types. Gene expression values are colored from green (low expression) to red (high expression). a: Patient expression data hierarchically clustered b: Patient expression data grouped by cancer type. Clusters of patients and genes are labelled

extremely abundant in breast, esophageal, head \& neck, lung, and stomach cancers compared to the other tumor types.

\section{MMP11 and MMP13 are nearly universally upregulated in} cancer

MMP11 and MMP13 expression was dramatically higher in most cancer types compared to tissue matched controls (Fig. 3). Aside from KIRP and PRAD for $M M P 13$, and $\mathrm{KICH}$ and UCEC for MMP11, the upregulation was consistent across all cancer types. MMP11 expression was comparatively high in the normal UCEC tissue, while the other non-significant pairs retained relatively low expression within the cancer samples. Both genes typically featured a very high fold change difference between groups and were among the highest expression differences observed within the dataset (Fig. 1).

\section{Diagnostic value of MMPs for Cancer}

The area under the curve (AUC) values for ROC analysis of MMP expression in each cancer are illustrated in Fig. 4a. Values for the range of the AUC as well as the sensitivity values at 90, 95, 99, and $100 \%$ specificity are included in Additional file 1: Table S1 for gene-cancer pairs with the highest AUC values. Each cancer type featured at least one MMP with an AUC greater than 0.9 except for PRAD. Six cancer types (BRCA, COAD, ESCA, HNSC, LUSC, and UCEC) showed 4 or more MMPs with AUC greater than 0.9. MMP11 most frequently had significant predictive values, with AUC greater than 0.9 in twelve cancers and greater than 0.95 in eight. The highest AUC observed for MMP11 was in LUSC with an AUC value of 0.991 (Fig. 4b). Seven MMPs (MMP7, MMP11, $M M P 12, M M P 13, M M P 24, M M P 27$, and $M M P 28)$ had an AUC of greater than 0.95 for at least one cancer type. For example, MMP12 had an AUC of 0.983 in lung squamous cancer (LUSC) (Fig. 4b).

\section{Combinations of dysregulated MMPs strongly predictive of cancer}

Using a multivariate analysis, ROC curves were made analyzing expression data using combinations of up to five MMPs for each cancer type. Additional file 1: Figure S1 displays AUC graphs for the top two individual MMPs and combinations of MMPs. The AUC for a combination was usually higher than with a single gene, however was not necessarily superior, as many of the confidence intervals for individual MMPs overlapped with the AUC value of MMPs in combination (Additional file 1: Table S1). For example, for thyroid cancer (THCA), MMP11 showed an 


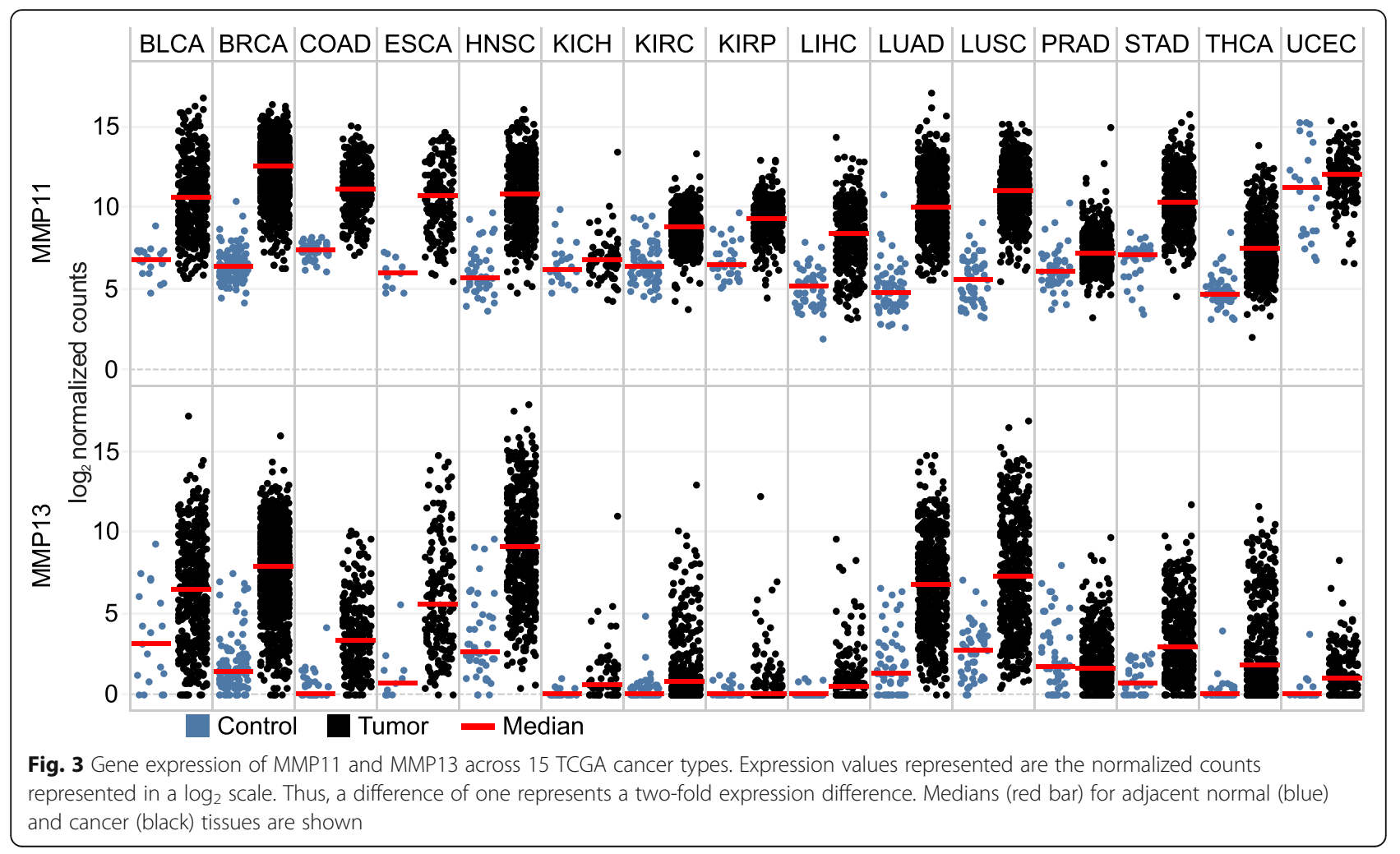

$4 \mathrm{~A}$

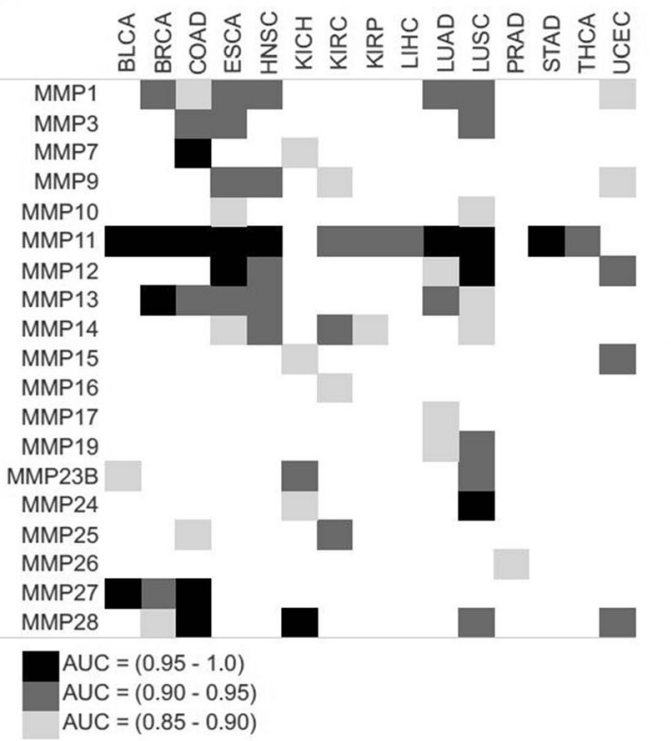

4B

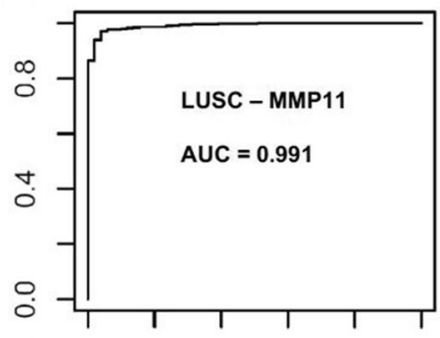

4C

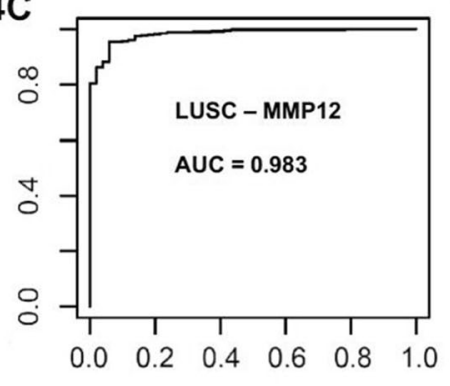

4D
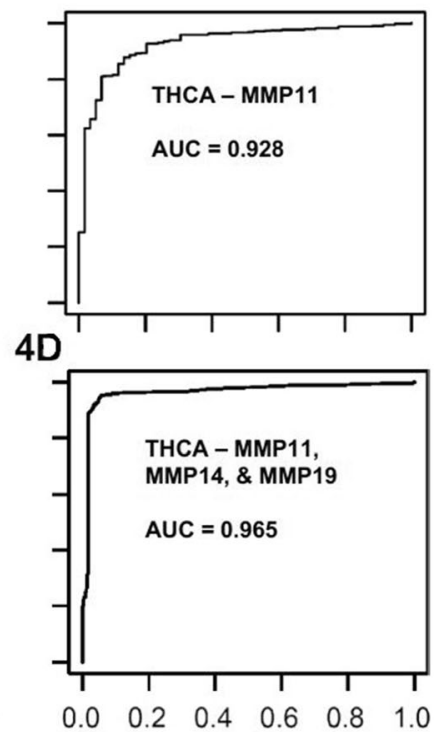

Fig. 4 Area under the curve values for receiver operator characteristic (ROC) curves for MMPs across TCGA cancer types. a Summary of AUC values. $\mathbf{b}$ ROC curve for MMP11 in squamous lung cancer. c ROC curve for MMP12 in squamous lung cancer. $\mathbf{d}$ Comparison of univariate analysis of the ROC curve for MMP11 (the MMP with the highest AUC value for thyroid cancer) and multivariate analysis in thyroid cancer combining expression patterns from MMP11, MMP14, and MMP19 
AUC of 0.928 alone, but when combined with MMP19, multivariate analysis showed an AUC of 0.961 (Fig. 4c). In colon cancer (COAD), an AUC of 1 was achieved after multivariate analysis of a combination of $M M P 7$, $M M P 13$, and $M M P 28$. The cancer type with the next highest AUC after multivariate analysis was lung squamous (LUSC). Individually, MMP11 and MMP12 were the MMPs with the best diagnostic value with an AUC of 0.991 and 0.983 (Fig. 4b), respectively for LUSC. After multivariate analysis two combinations of MMP11 and MMP12 yielded an AUC of 0.996.

\section{Prognostic value of MMPs for Cancer}

Twenty-four proteins from the MMP family were independently analyzed for their ability to predict overall survival in 15 different cancer types using tumor samples from TCGA. Each cancer type was considered independently when examining MMP prediction of overall survival. Hazard ratios, expression thresholds, and $p$-values for the thirty-seven curves that had $(p<0.01$ with $\mathrm{HR}$ or $1 / \mathrm{HR}>$ 1.5) are shown in Additional file 1: Table $S 2$ and summarized in Fig. 5a. Of these relationships, sixteen were in a type of renal cell carcinoma (KICH, KIRC, or KIRP). Liver cancer (LIHC) was the only other cancer type with three
MMPs associated with a survival change. Genes frequently associated with survival differences included MMP14, $M M P 17$, and $M M P 23 B$ which were associated with exclusively poorer prognosis for higher expression while $M M P 15, M M P 20$, and MMP24 were associated exclusively with poorer prognosis for lower expression. Interestingly, several MMPs featured inconsistent survival trends across cancer types with MMP19 showing opposite trends when comparing renal papillary (KIRP) with renal clear cell (KIRC) (Fig. 5b-c). The strongest hazard ratios were observed for MMP15 within renal papillary and renal chromophobe (KICH) subtypes (Fig. 5d-e).

\section{Discussion}

\section{MMP-11 is frequently upregulated in cancer}

The observation of MMP11 upregulation across cancer types is consistent with prior knowledge of gene function facilitating tumor invasion by degrading collagen, fibronectin, and laminin. Upregulated in 13 of 15 cancer types (Fig. 1), MMP11 expression differences yielded particularly high fold changes and high AUCs. For example, in lung cancers there was a 43-fold increase in squamous (LUSC) and 36-fold increase in adenocarcinoma (LUAD) over control. This upregulation of MMP11 was contrasted

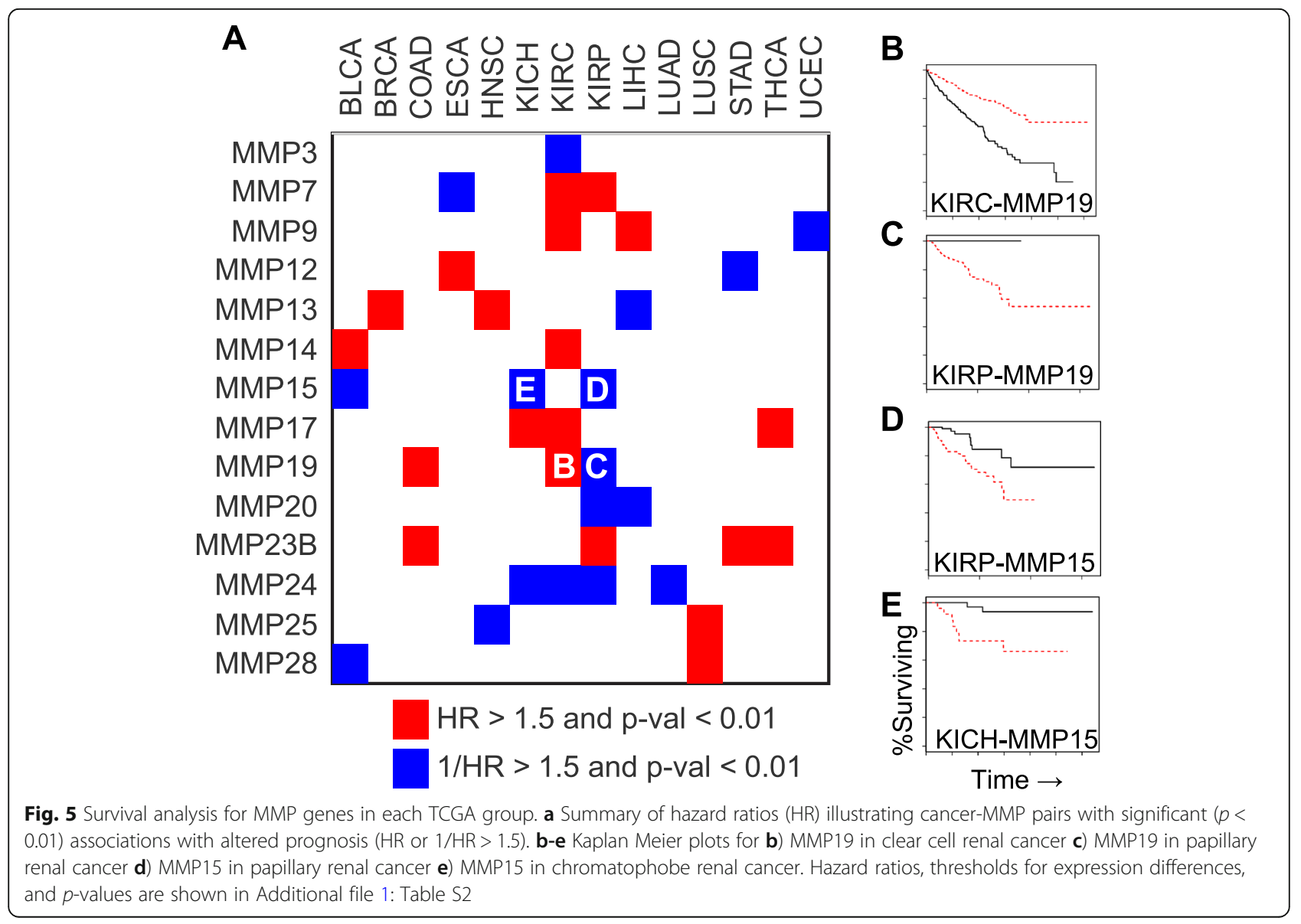


by other MMPs where up-regulation was more cancertype specific. A reason for this pan-cancer upregulation might be related to the functional ability of MMP-11 in helping evade immune surveillance by desensitizing cancer cells to NK-cells [38]. MMP-11 differs from other MMPs because it is not secreted as a pro-MMP, but is already active upon secretion through intracellular activation by furin, within the constitutive secretory pathway of the trans-Golgi network [39]. Our analysis supports a theory that MMP-11 expression is critical in cancer development and progression.

\section{MMP-7 is extremely upregulated in colon cancer}

MMP-7, a matrilysin protein, had a gene expression fold change of 141.85 in COAD, the highest fold change we found in our analysis. This is consistent with prior studies of colon cancer, that also have indicated that MMP-7 could predict a more aggressive phenotype of colon cancer and correlate negatively with patient survival [40-43]. However, TCGA data did not show any significant associations between survival and $M M P 7$ expression in colon cancer. MMP-7, a stromelysin, degrades collagens, proteoglycans, elastin, laminin, fibronectin, and casein. It also activates other MMPs, including gelatinase. Upregulation of $M M P 7$ was primarily found within GI cancers (esophageal, stomach, colon) as well as thyroid and head/neck. Though upregulated in other cancer types, the degree of upregulation for colon cancer, is truly remarkable with a fold change greater than 140. Part of the reason for this level of upregulation could be ascribed to the intrinsic expression of MMP7 in colon versus other tissues. Additionally, MMP-7 is very closely associated with some of the prime factors in the tumorigenesis of colon cancer. MMP-7 activation is directly associated with APC, and it is well established that APC mutations are frequently implicated as among the first mutations that occurs in the disease history of colon polyps as they progress to cancer [44]. APC mutations often lead to increased $\beta$-catenin and thus overexpression of MMP-7. If these changes occur in the earliest stages of colon cancer or precancer as we suspect, MMP-7 may be an excellent early diagnostic marker for colon cancer.

\section{Downregulation of MMPs}

Several MMPs that were largely downregulated in tumor versus control were membrane-type or unclassified by their primary degradation target. MMP-28, also known as epilysin, is downregulated in nine cancer types in our analysis (Fig. 1). This downregulation was highly pronounced in both lung squamous and adenocarcinoma subtypes. The finding is especially intriguing given that stable expression of MMP-28 in lung adenocarcinoma cells results in epithelial mesenchymal transition with accompanying loss of E-cadherin as reported by Illman et al. [37, 45]. Our analysis and prior reports do not capture a clear mechanistic or clinical picture how MMP-28 downregulation interplays with disease progression or metastasis.

\section{MMPs as diagnostic markers}

We believe that MMPs have tremendous potential as diagnostic biomarkers. Survival in cancer can be improved by diagnosis at an early stage. Screening tests such as colonoscopy plus biopsy, the gold standard for colon cancer diagnosis, are often invasive, costly, or uncomfortable while others such as a fecal occult blood test have a lower sensitivity. Identifying novel biomarkers for cancer screening hopefully will lead to highly sensitive, cost-effective, and non-invasive diagnostics. MMPs offer theoretical potential for these properties. Six cancer types featured four or more MMPs with AUC $>0.9$ (Fig. 4a). Cancer-by-cancer, lung squamous (LUSC) has two MMPs with sensitivities over 95\% (MMP11 and MMP12), colon cancer (COAD) has three over 94\% (MMP11, MMP28, and MMP7), and esophageal cancer (ESCA) has two over 94\% (MMP11 and $M M P 12)$. MMP11 was $100 \%$ specific and over $93 \%$ sensitive in colon and esophageal cancer. While, this data indicates that MMPs have the potential to serve as quality biomarkers, confirmation of upregulation in more easily accessed biofluids remains to be determined as increased gene expression does not necessarily mean a protein will be overexpressed at a high enough level to be detected within a biofluid, such as serum, at a high enough level to be acted upon. Will be needed to fulfill the qualities of an excellent, minimally-invasive biomarker. If serum detection is achievable, ubiquitously upregulated MMPs such as MMP11 or MMP13 could serve as a pan-cancer marker, capable of identifying the presence of cancer but not the specific location of the cancer.

\section{MMP combinations as diagnostic markers}

Several MMPs interact with each other or rely on other MMPs for their activation. For example, the activation pathway of pro-MMP-2 is cleaved by MMP-14 [2]. It is likely that the dysregulation of one MMP alters the MMP ecosystem and means that MMPs are better predictors when analyzed in combination as opposed to individually. Simultaneous upregulation of MMPs that cleave the same substrates were frequently observed within our data. The combined signal of MMP11 and $M M P 19$, both of which cleave aggrecan and gelatin [46], resulted in a higher AUC value than individually in thyroid cancer. Additionally, stromelysins and collagenases both showed similar patterns of upregulation across tumor types. The inter-dependence and similar functions of MMPs give physiological rationale for performing multivariate analysis when observing MMP dysregulation. 


\section{MMPs as prognostic markers in kidney clear cell carcinoma}

MMPs are generally not great prognostic biomarkers for most cancer types. However, MMPs have very good prognostic value for kidney clear cell carcinoma (KIRC) (Fig. 5). High expression of MMP7, MMP9, MMP14, $M M P 17$ and MMP19 each individually correlated with a significantly poorer survival in patients with KIRC. Pathologic spread of these tumors from the renal cortex to the renal vein is facilitated by the lack of connective tissue between the renal columns and the vasculature in the renal sinus. Further degradation of the extracellular matrix from higher expression of MMPs could allow invasion of the renal vein and therefore more metastases earlier in the disease course. MMP-2 and -9 are reported in literature to be associated with poor prognosis in kidney clear cell carcinoma [47]. Both MMP-2 and MMP-9 are implicated in angiogenesis which is critical for highly vascularized malignancies such as renal cell carcinoma. Higher expression of MMP-2 and MMP-9 are found in patients who have kidney clear cell carcinoma metastases when compared to patients without distant metastases [48]. Other MMPs, especially those less studied or of lower abundance, may also have critical roles in the promotion of invasion, angiogenesis, and metastasis within this disease state.

The MMP dysregulation in renal cancer might be linked to VHL. VHL mutation, whether inherited or sporadic is frequently implicated in the tumorigenesis of renal clear cell carcinoma. With the loss of VHL, HIF is no longer properly degraded and free to induce the expression of proteins needed in hypoxic conditions. This leads to the upregulation of MMP14 in kidney clear cell carcinoma in patients with deletion of VHL [49]. We found that patients with higher expression of MMP14 had generally poorer survival, consistent with the more aggressive and invasive malignancy associated with VHL functional loss. Additionally, VHL regulates the assembly of collagen IV, a major constitute of the basement membrane and target of MMP-3, MMP-8, and MMP-9 [50]. This profile likely results in increased destruction and invasion through the basement membrane and may explain why higher expression of MMP-3 correlates with a poorer prognosis in kidney clear cell carcinoma patients [51]. Our evidence corroborates the literature knowledge of the significant role of MMPs in the disease history of kidney cancer.

\section{Conclusions}

This study had a number of strengths, including that it is the largest MMP gene expression analysis to date. It encompassed all MMP genes using RNA-seq technology across 15 different types of cancers. This manuscript explored both the significance of MMP expression in comparison to normal tissue expression and observed how MMP levels associate with survival differences. However, this manuscript had some limitations as it relates to the TCGA. One limitation was that based on information from the TCGA, it was not feasible for us to correlate how a tumor's stroma and supporting cells contribute to MMP expression. Other limitations include that we did not perform any analyses which correalated MMP expression to demographic or pathologic factors outside of tumor histological subtype. Despite these limitations, the findings within this manuscript are still relevant.

The information gained from this analysis support that several MMPs are almost ubiquitously upregulated across different cancer types while others are more specific to certain cancers. Based on the information presented in this manuscript, we believe the most actionable point is an investigation into the minimally invasive diagnostic capabilities of the MMPs which were the most ubiquitously upregulated across cancers such as MMP11 and MMP13. Functional studies or further exploration of these genes as potential pan-cancer biomarkers may provide a sizeable benefit to the medical community.

\section{Additional file}

Additional file 1: Figure S1. Top univariate and multivariate ROC curves for MMP-cancer pairs. Table S1. Sensitivity values for high AUC MMPcancer pairs. Table S2. Significant survival differences associated with altered MMP expression. (PDF $1499 \mathrm{~kb}$ )

\section{Abbreviations}

BLCA: Bladder Cancer; BRCA: Breast Cancer; COAD: Colon Adenocarcinmoa; ECM: Extracellular Matrix; EMT: epithelial mesenchymal transition;

ESCA: Esophageal Cancer; FC: Fold Change; HIF: Hypoxia Induced Factor; HNSC: Head and Neck Cancer; HR: Hazard Ratio; KICH: Kidney Chromophobe; KIRC: Renal Clear Cell Cancer; KIRP: Renal Papillary Cancer;

LIHC: Hepatocellular Cancer; LUAD: Lung Adenocarcinoma; LUSC: Lung Squamous Cancer; MMP: Matrix Metalloproteases; PRAD: Prostate Adenocarcinoma; pval: $p$-value; ROC: Receiver Operator Characteristic; STAD: Stomach Adenocarcinoma; TCGA: The Cancer Genome Atlas;

THCA: Thyroid Cancer; UCEC: Uterine Corpus Endometrial Carcinoma; VHL: Von Hippel-Lindau

\section{Acknowledgements \\ Special thanks to the researchers that collected, curated, and maintain the TCGA data, whose high-quality work and effort have made studies like this possible.}

\section{Authors' contributions}

$E G, K B$, and JW, contributed to the drafting of the manuscript, conception, analysis and interpretation, data representation. DM, SS, and NS, contributed to the conception, analysis and interpretation. SB, and AS, contributed to the data analysis and statistical analysis. RS, contributed to the data analysis, statistical analysis, data representation, analysis and interpretation. JXS, contributed to the drafting, conception, analysis and interpretation, supervision, funding, and guidance. All authors have read and approved of this manuscript and contributed to it. 


\section{Funding}

Research was funded through funding by the Georgia Research Alliance of Jin-Xiong She as an Eminent Scholar. This research funding was used to fund all aspects of the study including design, data collection, and data analysis.

\section{Availability of data and materials}

Raw data was obtained from TCGA repositories and were obtained for our purposes from (https://xenabrowser.net/datapages/). Analyzed data is available within supplementary information or from the authors upon reasonable request.

\section{Ethics approval and consent to participate}

Data was collected from public data repositories.

\section{Consent for publication}

Not Applicable.

\section{Competing interests}

The authors declare that they have no competing interests.

Received: 6 September 2018 Accepted: 29 May 2019

Published online: 14 June 2019

\section{References}

1. Gialeli C, Theocharis AD, Karamanos NK. Roles of matrix metalloproteinases in cancer progression and their pharmacological targeting. FEBS J. 2011;278(1):16-27.

2. Jobin PG, Butler GS, Overall CM. New intracellular activities of matrix metalloproteinases shine in the moonlight. Biochim Biophys Acta Mol Cell Res. 2017;1864(11 Pt A):2043-55

3. Egeblad M, Werb Z. New functions for the matrix metalloproteinases in cancer progression. Nat Rev Cancer. 2002;2(3):161-74.

4. Vihinen P, Kahari VM. Matrix metalloproteinases in cancer: prognostic markers and therapeutic targets. Int J Cancer. 2002;99(2):157-66.

5. Liu $\mathrm{H}$, et al. The role of MMP-1 in breast cancer growth and metastasis to the brain in a xenograft model. BMC Cancer. 2012;12:583.

6. Kessenbrock K, Plaks V, Werb Z. Matrix metalloproteinases: regulators of the tumor microenvironment. Cell. 2010;141(1):52-67.

7. Lederle $\mathrm{W}$, et al. MMP13 as a stromal mediator in controlling persistent angiogenesis in skin carcinoma. Carcinogenesis. 2010;31(7):1175-84.

8. Wang $F Q$, et al. Matrilysin (MMP-7) promotes invasion of ovarian cancer cells by activation of progelatinase. Int J Cancer. 2005;114(1):19-31.

9. Zhao $Y$ G, et al. Activation of pro-gelatinase B by endometase/matrilysin-2 promotes invasion of human prostate cancer cells. J Biol Chem. 2003;278(17):15056-64

10. Ahokas $\mathrm{K}$, et al. Matrilysin-2 (matrix metalloproteinase-26) is upregulated in keratinocytes during wound repair and early skin carcinogenesis. J Invest Dermatol. 2005;124(4):849-56.

11. Decock J, et al. Matrix metalloproteinases: protective roles in cancer. J Cell Mol Med. 2011;15(6):1254-65.

12. Yang $\mathrm{H}$, et al. Knockdown of RHOC by shRNA suppresses invasion and migration of cholangiocellular carcinoma cells via inhibition of MMP2, MMP3, MMP9 and epithelial-mesenchymal transition. Mol Med Rep. 2016;13(6):5255-61.

13. Huang JF, Du WX, Chen JJ. Elevated expression of matrix metalloproteinase3 in human osteosarcoma and its association with tumor metastasis. J BUON. 2016;21(1):235-43.

14. Spalding TJ, et al. Fine needle aspiration cytology as part of a three stage diagnostic approach to breast lumps in a district general hospital. J R Nav Med Serv. 1989;75(1):5-9.

15. Stetler-Stevenson WG, Yu AE. Proteases in invasion: matrix metalloproteinases. Semin Cancer Biol. 2001;11(2):143-52.

16. McCawley LJ, Matrisian LM. Matrix metalloproteinases: multifunctional contributors to tumor progression. Mol Med Today. 2000;6(4):149-56.

17. Detry B, et al. Matrix metalloproteinase-2 governs lymphatic vessel formation as an interstitial collagenase. Blood. 2012;119(21):5048-56.

18. Morgunova $E$, et al. Structure of human pro-matrix metalloproteinase-2: activation mechanism revealed. Science. 1999;284(5420):1667-70.

19. Van den Steen PE, et al. Biochemistry and molecular biology of gelatinase $B$ or matrix metalloproteinase-9 (MMP-9). Crit Rev Biochem Mol Biol. 2002;37(6):375-536.
20. Verma RP, Hansch C. Matrix metalloproteinases (MMPs): chemical-biological functions and (Q)SARs. Bioorg Med Chem. 2007;15(6):2223-68.

21. Vaananen A, et al. Expression of collagen XVIII and MMP-20 in developing teeth and odontogenic tumors. Matrix Biol. 2004;23(3):153-61.

22. Remacle $A G$, et al. Selective function-blocking monoclonal human antibody highlights the important role of membrane type-1 matrix metalloproteinase (MT1-MMP) in metastasis. Oncotarget. 2017:2781.

23. Cepeda MA, et al. Inhibition of MT1-MMP proteolytic function and ERK1/2 signalling influences cell migration and invasion through changes in MMP-2 and MMP-9 levels. J Cell Commun Signal. 2017:167-79.

24. Liu Y, et al. MT2-MMP induces proteolysis and leads to EMT in carcinomas. Oncotarget. 2016;7(30):48193-205.

25. Itoh Y. Membrane-type matrix metalloproteinases: their functions and regulations. Matrix Biol. 2015;44-46:207-23.

26. Tatti $\mathrm{O}$, et al. MMP16 mediates a proteolytic switch to promote cell-cell adhesion, collagen alignment, and lymphatic invasion in melanoma. Cancer Res. 2015:75(10):2083-94.

27. Lin F, et al. Inhibitory effects of miR-146b-5p on cell migration and invasion of pancreatic cancer by targeting MMP16. J Huazhong Univ Sci Technolog Med Sci. 2011;31(4):509-14.

28. Sohail A, et al. MT4-(MMP17) and MT6-MMP (MMP25), a unique set of membrane-anchored matrix metalloproteinases: properties and expression in cancer. Cancer Metastasis Rev. 2008;27(2):289-302.

29. Llano $E$, et al. Identification and characterization of human MT5-MMP, a new membrane-bound activator of progelatinase a overexpressed in brain tumors. Cancer Res. 1999;59(11):2570-6.

30. Takino T, et al. Cleavage of metastasis suppressor gene product KiSS-1 protein/metastin by matrix metalloproteinases. Oncogene. 2003;22(30):4617-26.

31. Sun Q, et al. MMP25 (MT6-MMP) is highly expressed in human colon cancer, promotes tumor growth, and exhibits unique biochemical properties. J Biol Chem. 2007:282(30):21998-2010.

32. Beck IM, et al. MMP19 is essential for T cell development and T cellmediated cutaneous immune responses. PLoS One. 2008;3(6):e2343.

33. $\mathrm{Yu} \mathrm{G}$, et al. Matrix metalloproteinase-19 promotes metastatic behavior in vitro and is associated with increased mortality in non-small cell lung cancer. Am J Respir Crit Care Med. 2014;190(7):780-90.

34. Bister $V$, et al. Increased expression of matrix metalloproteinases-21 and -26 and TIMP-4 in pancreatic adenocarcinoma. Mod Pathol. 2007;20(11):1128-40.

35. Riddick AC, et al. Identification of degradome components associated with prostate cancer progression by expression analysis of human prostatic tissues. Br J Cancer. 2005;92(12):2171-80.

36. Chinello $C$, et al. Tumor size, stage and grade alterations of urinary peptidome in RCC. J Transl Med. 2015;13:332.

37. IIIman SA, Lohi J, Keski-Oja J. Epilysin (MMP-28)--structure, expression and potential functions. Exp Dermatol. 2008;17(11):897-907.

38. Nagase $H$, Visse R, Murphy G. Structure and function of matrix metalloproteinases and TIMPs. Cardiovasc Res. 2006;69(3):562-73.

39. Matziari M, Dive V, Yiotakis A. Matrix metalloproteinase 11 (MMP-11; stromelysin-3) and synthetic inhibitors. Med Res Rev. 2007;27(4):528-52.

40. Fang $Y$, et al. Elevated expressions of MMP7, TROP2, and survivin are associated with survival, disease recurrence, and liver metastasis of colon cancer. Int J Color Dis. 2009:24(8):875

41. Kurokawa $\mathrm{S}$, et al. Tumour matrilysin expression predicts metastatic potential of stage I (pT1) colon and rectal cancers. Gut. 2005;54(12):1751-8.

42. Zeng Z-S, et al. Matrix Metalloproteinase-7 expression in colorectal Cancer liver metastases. Clin Cancer Res. 2002;8(1):144-8.

43. Fuksiewicz $M$, et al. The matrix metalloproteinase-7 and pro-enzyme of metalloproteinase-1 as a potential marker for patients with rectal cancer without distant metastasis. Tumor Biol. 2015;36(5):3629-35.

44. Gustavson MD, et al. Tcf binding sequence and position determines $\beta$ catenin and Lef-1 responsiveness of MMP-7 promoters. Mol Carcinog. 2004;41(3):125-39.

45. IIIman SA, et al. Epilysin (MMP-28) induces TGF- $\beta$ mediated epithelial to mesenchymal transition in lung carcinoma cells. J Cell Sci. 2006:119(18):3856-65.

46. Chakraborti $S$, et al. Regulation of matrix metalloproteinases: an overview. Mol Cell Biochem. 2003;253(1-2):269-85.

47. Kallakury BV, et al. Increased expression of matrix metalloproteinases 2 and 9 and tissue inhibitors of metalloproteinases 1 and 2 correlate with poor prognostic variables in renal cell carcinoma. Clin Cancer Res. 2001;7(10):3113-9. 
48. Slaton JW, et al. Expression levels of genes that regulate metastasis and angiogenesis correlate with advanced pathological stage of renal cell carcinoma. Am J Pathol. 2001;158(2):735-43.

49. Petrella BL, Lohi J, Brinckerhoff CE. Identification of membrane type-1 matrix metalloproteinase as a target of hypoxia-inducible factor-2a in von HippelLindau renal cell carcinoma. Oncogene. 2005;24(6):1043-52.

50. Kurban $\mathrm{G}$, et al. Collagen matrix assembly is driven by the interaction of von Hippel-Lindau tumor suppressor protein with hydroxylated collagen IV alpha 2. Oncogene. 2008;27(7):1004-12.

51. Radisky DC, et al. Rac1b and reactive oxygen species mediate MMP-3induced EMT and genomic instability. Nature. 2005;436(7047):123-7.

\section{Publisher's Note}

Springer Nature remains neutral with regard to jurisdictional claims in published maps and institutional affiliations.

Ready to submit your research? Choose BMC and benefit from:

- fast, convenient online submission

- thorough peer review by experienced researchers in your field

- rapid publication on acceptance

- support for research data, including large and complex data types

- gold Open Access which fosters wider collaboration and increased citations

- maximum visibility for your research: over $100 \mathrm{M}$ website views per year

At $B M C$, research is always in progress.

Learn more biomedcentral.com/submissions 